\title{
Estudo dos limiares de audibilidade nas altas frequências em trabalhadores da área odontológica
}

\section{A study of the high-frequency hearing thresholds of dentistry professionals}

\author{
Andréa Cintra Lopes ${ }^{1}$, Ana Dolores Passarelli de Melo², Cibele Carmelo Santos³.
}

1) Doutor. Professor Doutor.

2) Mestre em Ciências da Comunicação Humana pela Faculdade de Odontologia de Bauru - USP. Fonoaudióloga Clínica Spazio Fonoaudiológico, Lima - Peru.

3) Mestre em Ciências da Comunicação Humana pela Faculdade de Odontologia de Bauru - USP. Fonoaudióloga da AMA - Aparelhos Auditivos e CPAP.

Instituição: Faculdade de Odontologia de Bauru - Universidade de São Paulo

Bauru / SP - Brasil.

Endereço para correspondência: Andréa Cintra Lopes - Alameda Dr. Octávio Pinheiro Brizolla, 9-75 - Departamento de Fonoaudiologia - Vila Universitária - Bauru / SP

- Brasil - CEP: 17012-901 - E-mail: aclopes@usp.br

Pesquisa financiada pelo órgão de fomento FAPESP, porcesso n. 2007/01074-7.

Artigo recebido em 30 de setembro de 2011. Artigo aprovado em 26 de outubro de 2011.

\section{RESUMO}

Introdução: Na prática odontológica, o Cirurgião-Dentista está sujeito aos efeitos nocivos provocados por diversos agentes, como pelo ruído emitido por seus instrumentos de trabalho. Em 1959, a American Dental Association recomendava avaliações audiométricas periódicas e uso de proteção auditiva. São necessárias maiores informações sobre o comportamento auditivo dessa população: Cirurgiões-Dentistas, Auxiliares e Protéticos para se propor medidas de prevenção e tratamento precoce.

Objetivo: Investigar os limiares de audibilidade em Cirurgiões-Dentistas, Auxiliares e Protéticos.

Método: Forma de estudo: Estudo Clínico.Participaram 108 profissionais, sendo 44 Cirurgiões-Dentistas (GI), 36 Auxiliares (G II) e 28 Protéticos (GIII). Foram realizadas: entrevista específica, meatoscopia, audiometria tonal convencional e de altas frequências, logoaudiometria, imitanciometria.

Resultados: A comparação entre as médias dos limiares evidenciaram piora com o aumento da frequência para os 3 grupos testados; para a média tritonal de 500 a $2000 \mathrm{~Hz}$, e 3000 a 6000 $\mathrm{Hz}$, o GIII apresentou os piores limiares, já para a média das altas frequências $(9000 \mathrm{a} 16.000 \mathrm{~Hz})$ o GII apresentou os piores limiares.

Conclusão: A avaliação audiológica convencional não identificou exames alterados para os três grupos testados, no entanto, o exame da avaliação audiológica complementar como a audiometria de altas frequências indicou maior sensibilidade na detecção precoce de alterações auditivas uma vez que a perda auditiva dessa população acomete as frequências que não são testadas nos exames convencionais. Dessa maneira enfatizamos nesse trabalho a necessidade de inserir na rotina de exames a audiometria de altas frequências juntamente com os outros exames audiológicos.

Palavras-chave: audiometria, perda auditiva de alta frequência, ruído, odontologia geral.

\section{SUMMARY}

Introduction: In the dentistry practice, dentists are exposed to harmful effects caused by several factors, such as the noise produced by their work instruments. In 1959, the American Dental Association recommended periodical hearing assessments and the use of ear protectors. Aquiring more information regarding dentists', dental nurses', and prosthodontists' hearing abilities is necessary to propose prevention measures and early treatment strategies.

Objective: To investigate the auditory thresholds of dentists, dental nurses, and prosthodontists.

Method: In this clinical and experimental study, 44 dentists (Group I; GI), 36 dental nurses (Group II; GII), and 28 prosthodontists (Group III; GIII) were included, , with a total of 108 professionals. The procedures that were performed included a specific interview, ear canal inspection, conventional and high-frequency threshold audiometry, a speech reception threshold test, and an acoustic impedance test.

Results: In the 3 groups that were tested, the comparison between the mean hearing thresholds provided evidence of worsened hearing ability relative to the increase in frequency. For the tritonal mean at 500 to $2,000 \mathrm{~Hz}$ and 3,000 to 6,000 Hz, GIII presented the worst thresholds. For the mean of the high frequencies $(9,000$ and 16,000 Hz), GII presented the worst thresholds.

Conclusion: The conventional hearing threshold evaluation did not demonstrate alterations in the 3 groups that were tested; however, the complementary tests such as high-frequency audiometry provided greater efficacy in the early detection of hearing problems, since this population's hearing loss impaired hearing ability at frequencies that are not tested by the conventional tests. Therefore, we emphasize the need of utilizing high-frequency threshold audiometry in the hearing assessment routine in combination with other audiological tests. Keywords: audiometry, hearing loss, high-frequency, hearing loss, noise-induced, dentistry. 


\section{INTRODUÇÃO}

O ruído é um dos mais significantes agentes nocivos à saúde, principalmente à audição, e está presente não só em ambientes de trabalho como também de lazer. Nas doenças adquiridas no ambiente de trabalho, a perda auditiva induzida por ruído (PAIR), ou perda auditiva induzida por níveis de pressão sonora elevada (PAINPSE) é uma das mais comuns.

Atualmente, o ruído faz parte do cotidiano das pessoas, está presente no trânsito, no lazer, assim como no trabalho, sendo assim, a PAIR poderá ser uma das principais doenças crônicas no futuro da humanidade (FIORINI, 2000).

A PAIR é definida como perda auditiva sensorioneural, decorrente da exposição ocupacional sistemática a níveis de pressão sonora elevado, ocasionando lesão nas células ciliadas do Órgão de Corti. Em geral, ébilateral e simétrica, insidiosa e irreversível, está diretamente relacionada ao tempo de exposição e susceptibilidade individual. (Comitê Nacional de Ruído e Conservação Auditiva, 1999; Rabinowitz, 2000). Essa alteração auditiva manifesta-se, primeiramente nas frequências de $4000 \mathrm{~Hz}, 6000 \mathrm{~Hz}$ e $3000 \mathrm{~Hz}$ e estendese, com a progressão, às frequências de $8000 \mathrm{~Hz}, 2000 \mathrm{hz}$, $1000 \mathrm{~Hz}, 500 \mathrm{~Hz}$ e $250 \mathrm{~Hz}$. Raramente o ruído leva à perda auditiva profunda, e em geral, não ultrapassa $75 \mathrm{~dB}$ para altas frequências e $40 \mathrm{~dB}$ nas baixas frequências, atingindo seu máximo nos primeiros 10 a 15 anos de exposição (Luxon, 1998, Hanger, Barbosa-Branco, 2004, Gatto et al, 2005).

Por apresentar um surgimento dos sintomas auditivos tardios e insidiosos, os profissionais geralmente só perceberão a dificuldade auditiva quando a lesão já está em estágio avançado (SAVA, 2005). A exposição contínua a sons com níveis elevados pode acarretar, além do dano auditivo, algumas alterações secundárias, como zumbido, estresse, alterações fisiológicas no ritmo cardíaco e na pressão sanguínea, bem como dificuldade na discriminação de sons da fala, principalmente em ambientes ruidosos. O ruído provoca exaustão física, alterações químicas, metabólicas e mecânicas do órgão sensorial auditivo, tendo como resultado final uma perda auditiva parcial ou total do órgão de Corti, parte interna do ouvido (OtONi A, BOger ME, BarbosaBranco A, Shimizu He, Maftum, MA, 2008).

O cirurgião dentista, basicamente está exposto a dois tipos de ruído: o ruído externo ao seu ambiente de trabalho e ao ruído proveniente dos seus equipamentos de trabalho, tais como o ruído provocado pelo motor de alta e baixa rotação, compressor, ar condicionado, amalgamador, sugador entre outros. (Hinze, Deleon e Mitchel, 1999) Além do ruído, ficam expostos a outros agentes, entre eles: químicos (substâncias manipuladas, principalmente o mercúrio); biológicos (a cavidade oral é rica em microorganismos, assim o risco de contagio de doenças como a hepatite e a AIDS se torna maior); mecânicos (lesões corporais ou perfurantes pelos instrumentos utilizados); sociais (trabalho que envolve muita tensão e exige um domínio da situação para facilitar o relacionamento paciente/profissional), e ergonômicos (em função da postura de trabalho o profissional está sujeito a problemas de coluna, articulações do braço e varizes) (SouzA, 1997). De acordo com PRESTA et al. (2004) os profissionais da área odontológica têm apresentado com frequência desconfortos relacionados ao desempenho profissional, os quais podem progredir chegando a lesões por esforços repetitivos (LER) ou distúrbios osteomusculares relacionados ao trabalho (DORT).

A American Dental Association (ADA), em 1959 já recomendava avaliações audiológicas periódicas nos cirurgiões dentistas, devido à exposição prolongada a sons intensos, provocados por instrumentos como brocas de alta velocidade, sistemas de ejeção, máquinas ultra-sônicas, cortadores de modelos, equipamentos de alta velocidade de sucção e vibração, o que pode levar a perda auditiva. Estudos (Altinoz et al. (2001); Fernandes et al. (2004)) que realizaram medições do nível de ruído no ambiente de trabalho dos cirurgiões dentistas observaram níveis de ruído superiores a 80 dB NPS.

No Brasil, a Lei 6.514 de 22 de dezembro de 1977 da Consolidação de Leis do Trabalho, relaciona os parâmetros que proporcionam o conforto acústico dos trabalhadores juntamente com as normas da Associação Brasileira de Normas Técnicas (ABNT), sendo que a legislação do trabalho brasileira definiu que o limite máximo de ruído tolerável é de 85 dB NPS em uma jornada de trabalho de 8 horas (Segurança e Medicina do Trabalho, 1991); a NBR 10.152, indica que para consultório odontológico esse valor deve situar entre 35 e $45 \mathrm{~dB}(\mathrm{~A})$.

Paraguay (1999) em seu estudo verificou que os cirurgiões dentistas com cinco ou mais anos de atuação profissional tiveram resultados alterados no exame de audiometria tonal limiar

LEGGAT (2000) relatou que a possibilidade de alguns cirurgiões dentistas com mais tempo de profissão sofrerem alterações auditivas pode ser devido a anteriores exposições a antigos equipamentos, o que não foi considerado neste estudo que a casuística era relativamente jovem.

Estudos (Oliveira et al. (2007), Torrês et al. (2007), Melo et al. (2008)) mostraram que os profissionais da classe odontológica devem ser conscientizados sobre o ruído ocupacional bem como sobre as consequências danosas 
que representam para a sua saúde. E essa conscientização, para o controle e a prevenção desses efeitos, deve começar precocemente, durante o curso de graduação, período em que o profissional está sendo formado, para que, consciente dos riscos a que está exposto, possa prevenílos, em vez de tentar suavizar ou tratar os problemas decorrentes deles.

No Brasil, o trabalho de prevenção das doenças ocupacionais e dos acidentes de trabalho teve inicio de 1943, com a Consolidação da Leis do trabalho (CLT) e desde então os cuidados com a audição do trabalhador que atua em ambientes ruidosos é intensificada. Com a promulgação da Portaria nº 3.214/78 houve um importante avanço no âmbito da conservação auditiva, essa portaria, por meio da NR-7, torna obrigatória a realização da audiometria tonal liminar.

O interesse pelo diagnóstico precoce tem aumentado e considerando o desenvolvimento da tecnologia voltada a o diagnóstico da deficiência auditiva, assim como a presença de alterações auditivas na ausência de queixas e que não são comumente identificadas na audiometria tonal liminar, outros métodos têm sido utilizados para identificar precocemente as alterações auditivas.

De acordo com estudos já realizados a Audiometria Tonal de altas frequências (entre 9.000 e $18.000 \mathrm{~Hz}$ ) constitui um instrumento para o diagnóstico precoce de alterações auditivas decorrentes da exposição ao ruído (Porto et al. (2004), Lopes e Godoy (2006), Amorin et al. (2008)). Trabalhos Mota (2002), PorTo et al (2004) realizados para a investigação da audição em frequências convencionais e altas frequências na área odontológica tem mostrado uma tendência a rebaixamento dos limiares, ou seja, sugerindo predisposiçãoà alteração auditiva mais significativa com o tempo. Conforme o aumento da frequência, da idade e tempo de exposição houve queda maior da acuidade auditiva. Observaram maior incidência de perda nas frequências de $6 \mathrm{kHz}$ e $14 \mathrm{kHz}$.

Lopes e GODOy (2006) compilaram a literatura referente à contribuição e importância da audiometria de altas frequências (AT-AF), na identificação precoce da perda auditiva induzida por ruído. Demonstraram por meio de revisão, que somente a audiometria tonal convencional pode não ser eficaz na prevenção e identificação precoce da PAIR, além de descrever as variáveis metodológicas para sua realização. As autoras também sugerem este método como rotina no Programa de Prevenção de Perdas Auditivas Ocupacionais. Dessa forma, o objetivo desse estudo foi investigar os limiares de audibilidade nas frequências convencionais e altas frequências possibilitando uma prevenção precoce quanto à saúde auditiva dessa população.

\section{MÉTODO}

Este trabalho foi iniciado após a a provação do Comitê de Ética em Pesquisa da Faculdade de Odontologia de Bauru da Universidade de São Paulo, processo número 043/2007. Esta pesquisa foi financiada pelo órgão de fomento FAPESP sob o número de processo 2007/01074-7.

Foi um estudo com delineamento transversal em amostra composta por 108 participantes voluntários da comunidade de Bauru, subdivididos em três grupos experimentais, sendo o Grupo I (G I) formado por 44 cirurgiões dentistas (16 do gênero masculino e 28 feminino) com variação de idade entre 23 e 57 anos (média de 34 anos de idade), Grupo II (G II) constituído por 36 auxiliares, com variação de idade entre 21 e 59 anos (média de 38 anos de idade), todas do gênero feminino; e Grupo III por 28 protéticos (17 do gênero masculino e 11 feminino) com variação de idade entre 17 e 53 anos (média de 35 anos de idade) da área de odontologia.

Foram convidados a participar os profissionais de clínicas odontológicas e laboratórios particulares, bem como das Universidades e Faculdades da cidade de Bauru - SP - Brasil que ofereciam o curso de Odontologia, e de hospitais que apresentassem esses profissionais no corpo clínico. Primeiramente, todos os participantes foram esclarecidos sobre os objetivos deste estudo, o qual foi iniciado após a concordância e a assinatura do Termo de Consentimento Livre e Esclarecido.

Como critério de inclusão e exclusão foram considerados para este estudo apenas profissionais da área odontológica com no mínimo dois anos de experiência, e que não apresentassem nenhuma doença pré-existente como caxumba, hipertensão arterial, diabetes, meningite, HIV, sífilis, entre outras que possam comprometer a acuidade auditiva, e ou deficiência auditiva pré-existente.

Todos os indivíduos deste estudo foram submetidosà:

- Entrevista específica e meatoscopia: Realizada com o intuito de investigar as características individuais, tais como idade, tempo de trabalho na função, hábitos de lazer ruidosos, exposição a produtos químicos, além das condições de saúde e outras doenças que podem potencializar os efeitos dos riscos ambientais.

- Audiometria tonal liminar convencional (250 a 8.000 $\mathrm{Hz}$ ); Audiometria tonal liminar de altas frequências (9.000 a 16.000 Hz), e Logoaudiometria: Foram realizadas com o audiômetro SD 50, marca Siemens. Foi utilizado o tom warble para pesquisas dos limiares tonais, os quais foram apresentados por meio dos fones auriculares HDA 200. Para a pesquisa dos limiares tonais, utilizou-se a técnica descendente, sendo estabe- 
lecido o limiar auditivo em $50 \%$ das respostas positivas à detecção do som (Lopes e Godoy, 2006).

- Imitanciometria: A medida da imitância acústica e a pesquisa dos reflexos ipsilaterais e contralaterais do músculo estapédio foi realizada com o equipamento GSI Tymp Star. Foram classificadas de acordo com a proposta de JERGER (1970).

\section{RESULTADOS}

A análise dos dados da entrevista específica evidenciou que 65 participantes se incomodam com o ruído de trabalho, 50 dos participantes relataram dificuldades para compreensão da fala, 8 estiveram no serviço militar, 11 informaram a presença de trauma acústico, 32 informaram a exposição a produto químico e 35 informaram que estão expostos a ruído de lazer.

O Gráfico 1 apresenta média dos limiares de audibilidades das frequências avaliadas na orelha direita para todos os grupos.

A comparação da média dos limiares de audibilidade de cada frequência entre os três grupos avaliados foi realizada por meio do teste de Kruskall-Wallis, considerando significante pelo teste de Dunn. Assim, obteve-se diferença estatisticamente significante na orelha direita nas frequências de $2 \mathrm{kHz}$ ( $\mathrm{p}=, 0446$ ), $8 \mathrm{kHz}$ ( $\mathrm{p}=, 0492), 16 \mathrm{kHz}$ $(\mathrm{p}=, 0441)$ quando a média do Grupo I (média do limiar em $2 \mathrm{kHz} 5,91 \mathrm{~dB}$, em $8 \mathrm{kHz} 11,59 \mathrm{~dB}$ e em $16 \mathrm{kHz} 21,59 \mathrm{~dB}$ ) foi comparado ao do Grupo II (média do limiar em $2 \mathrm{kHz}$ 10,69 dB, em 8kHz 18,61 dB e em 16kHz 32,78 dB); portanto pode-se verificar que o GII apresentou estatisticamente piores limiares nas frequências de $2 \mathrm{kHz}, 8 \mathrm{kHz}$, $16 \mathrm{kHz}$ na orelha direita comparado ao GI.

É possível observar que a orelha direita e a orelha esquerda apresentaram configurações similares para a audiometria convencional e de altas frequências quando observados as médias dos limiares auditivos para todos os grupos, porém a orelha direita apresentou piores limiares auditivos do que quando a comparamos com a orelha esquerda (Gráfico 2).

Para a orelha esquerda, as frequências de $4 \mathrm{kHz}$ $(\mathrm{p}=, 0238)$ e $6 \mathrm{kHz}(\mathrm{p}=, 0310)$ apresentaram diferenças estatisticamente significante entre os limiares das referidas frequências entre o GI (média do limiar em $4 \mathrm{kHz} 8,41 \mathrm{~dB}$, em 6kHz 14,32 dB) e GII (média do limiar em 4kHz 14,03 dB, em 6kHz 20,69 dB) e, entre o GI e GIII (média do limiar em $4 \mathrm{kHz} 15,36 \mathrm{~dB}$, em $6 \mathrm{kHz} 22,32 \mathrm{~dB}$ ). Na frequência de $9 \mathrm{kHz}$ da orelha esquerda obteve-se diferença estatisticamente significante $(\mathrm{p}=0397)$ entre o GI (média $9 \mathrm{kHz}$ 10,91 dB) e GII (média do limiar em 9kHz 20,28 dB).

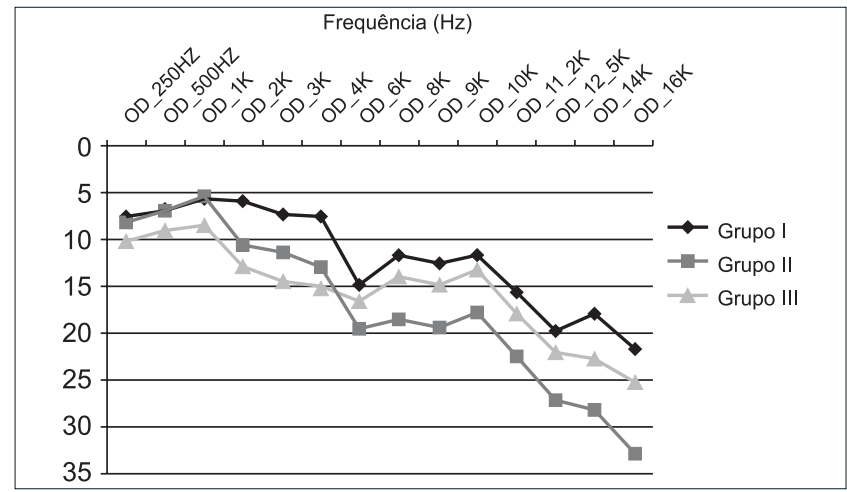

Gráfico 1. Limiares de audibilidade para orelha direita de todos os grupos.

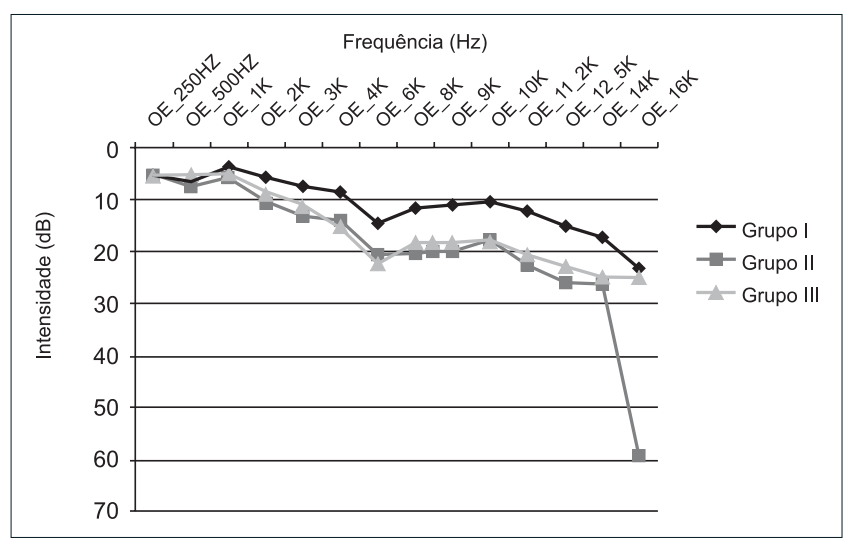

Gráfico 2. Limiares de audibilidade para a orelha esquerda de todos os grupos.

A comparação da média dos limiares de audibilidade das frequências de $500 \mathrm{~Hz}$ a $2 \mathrm{kHz}, 3 \mathrm{kHz}$ a $6 \mathrm{kHz}, 9 \mathrm{kHz}$ a $16 \mathrm{kHz}, 12 \mathrm{kHz}$ a $16 \mathrm{kHz}$, foi realizada por meio do Teste de Kruskall-Wallis, sendo considerado significância por meio do teste de Dunn. Para tal comparação obteve-se diferença estatisticamente significante $(\mathrm{p}=, 0147)$ apenas quando comparou-se a média dos limiares entre as frequências de $3 \mathrm{kHz}$ a $6 \mathrm{kHz}$ na orelha esquerda entre o G I (10 dB) e G II (15,93 dB), e G I (10 dB) e G III (16,25 dB).

A Logoaudiometria, realizada por meio do LRF confirmaram os resultados da audiometria convencional em 100\% dos participantes, assim como o IRF apresentou resultados compatíveis com os limiares audiométricos encontrados nos 100\% dos participantes.

Nas medidas da imitância acústica e pesquisa dos reflexos do músculo estapedio ipisilateral e contralateral foram obtidos registros de curva timpanométrica tipo A, bilateralmente em 100\% dos participantes, indicando, portanto que a orelha média não interferiu nos resultados obtidos. 


\section{DISCUSSÃO}

Esse estudo foi constituído por uma amostra de profissionais cirurgiões-dentistas, protéticos e auxiliares odontológicos com mais de dois anos de atuação na área Odontológica e com média de idade para os três grupos de 35 anos, sendo considerada uma amostra de adultos.

Nessa população a perda de audição pode acontecer devido à prolongada exposição a sons intensos produzidos por instrumento utilizado em seu uso diário o que traz prejuízo posteriormente à sua comunicação e qualidade de vida. Hinze, Deleon e Mitchel (1999).

Esse trabalho mostrou que somente $60 \%$ da amostra se incomodaram com o ruído de trabalho (Souza (1997), TôRREs et al. (2007), MELo et al. (2008)) e 43,3\% dos participantes relataram dificuldades para compreensão da fala enquanto que $32,4 \%$ informaram que estão expostos a ruído de lazer.

Esse estudo evidenciou que quando comparados as médias dos limiares auditivos de todos os grupos se mostraram piores com o aumento da frequência. MATTEWs et al. (1997), Beltrami (1999), Fernandes e Mota (2001), Mota (2002), Porto et al. (2004), Silva e Feitosa (2006), Lopes et al. (2006), Lopes e Godoy (2006), Lopes, Almeida, Zanconato e Mondelli (2007), Carvalho, Koga, Carvalho, IsHida (2007). Para todos os grupos tanto a audiometria convencional como a audiometria de altas frequências para cada orelha apresentaram configurações similares, porém ao compararmos ambas as orelhas para todos os grupos, observamos que a orelha direita apresentou média dos limiares auditivos piores do que as médias dos limiares auditivos da orelha esquerda. Concordando com o estudo de Zubick, Tolentino e Boffa (1980) e discordando do trabalho de GijBels et al. (2006) que coletaram dados sobre os efeitos na saúde ocupacional entre cirurgiões-dentistas e observaram que a perda de audição é maior no lado esquerdo para dentistas destro, o que poderia ser explicada pela pequena distância entre esta orelha e o giro / equipamentos de vibração para profissionais destros.

A média tritonal de 500 a $2000 \mathrm{~Hz}$, e 3000 a $6000 \mathrm{~Hz}$, nesse estudo, apresentou piores limiares para o grupo de protéticos (GIII), já para a média das altas frequências $(9000$ a $16.000 \mathrm{~Hz})$ o grupo dos profissionais auxiliares (GII) apresentou os piores limiares. Demonstrando a importância do exame complementar para a avaliação audiológica que nesse caso seria a audiometria de altas frequências que se utilizado como um exame de rotina clínica na avaliação desses profissionais irá colaborar para uma detecção precoce das alterações auditivas e prevenção.

\section{CONCLUSÃO}

Nesse estudo foi possível concluir que a avaliação audiológica convencional não identificou exames alterados para os três grupostestados, no entanto a identificação quanto aos limiares emaltas frequências indicaram comprometimento do sistema auditivo periférico, mais especificamente células ciliadas externas, portanto indicam maior sensibilidade na detecção precoce de alterações auditivas, favorecendo quanto a um exame complementar de prevenção.

\section{REFERÊNCIAS BIBLIOGRÁFIAS}

1. Altinoz HC, Gokbudak R, Bayraktar A, Belli SA. A pilot study of measurement of the frequency of sounds emitted by high-speed dental air turbines. J Oral Sci. 2001, 43(3):18992.

2. American Dental Association. Council on dental research. Sound hazard of high-peed cutting instumensts. Journal of the American Dental Associations. 1959, 58:145.

3. Amorin RB, Lopes AC, Santos KTP, Melo ADP, Lauris JRP. Alterações auditivas da exposição ocupacional em músicos. Arq. Int otorrinolaringol. 2008, 12(3):377-383.

4. Beltrami CHB. Dos limiares de audibilidade nas frequências de 250 a $18.00 \mathrm{~Hz}$ em indivíduos expostos a ruído ocupacional. Tese: Doutorado. Universidade Federal de São Paulo. 1999.

5. Brasil. Ministério do Trabalho - Secretaria da Segurança e Saúde no Trabalho. Portaria no. 19, de 09 de abril de 1998. diretrizes e parâmetros mínimos para a avaliação e acompanhamento da audição em trabalhadores expostos a níveis de pressão sonora elevados. Diário Oficial da União. Brasília, 22 de abril de 1998.

6. Comitê Nacional de Ruido e Conservação Auditiva. Perda auditiva induzida por ruído relacionada ao trabalho. Boletim, São Paulo, nº 1, 1999.

7. Carvalho RMM, Koga MC, De Carvalho M, Ishida IM. Limiares auditivos para altas frequencias em adultos sem queixa auditiva. ACTA ORL/Técnicas em Otorrinolaringologia. 2007, 25(1):62-6.

8. Fernandes JB, Mota HB. Estudo dos limiares de audibilidade nas altas frequências em trabalhadores expostos a ruído e solvente. Pró-fono. 2001, 13(1):1-8.

9. Fernandes JC, Ovileira JRE, Fernandes VM. Avaliação do ruídoem consultório odontológico.XISIMPEP. Bauru/SP. 2004. 
10. Fiorini AC. O uso de registro de emissões otoacústicas como instrumento de vigilância epidemiológica de alterações auditivas em trabalhadores expostos a ruído. [Tese, Doutorado em Saúde Ambiental] Faculdade de Saúde Pública, Universidade de São Paulo, São Paulo, 2000.

11. Gatto CI, Lermen RA, Teixeira TM, Magni C, Morata TC. A análise da conduta de médicos do trabalho diante de trabalhadores com perda auditiva. Rev Dist Com. 2005 17(1):101-15.

12. Gijbels et al. Potential occupational health problems for dentists in Flanders, Belgium. Clin Oral Invest Flanders. 2006, 10(1):8-16.

13. Hanger MRHC, Barbosa-Branco A. Efeitos auditivos decorrentes da exposição ocupacional ao ruído em trabalhadores de marmorarias no Distrito Federal. Rev Assoc Med Bras. 2004, 50(4):396-9.

14. Hinze H, Deleon C, Mitchel WC. Dentist at High Hisk for Hearing Loss: protection with custom earplugs. General Dentistry. 1999, 47(6):600-603.

15. Jerger J. clinical experience with impedance audiometry. Arch Otolaryngol. Chicago. 1970, 92(4):311-24.

16. Leggat PA, Chwanadisai S, Kukiattrakoon B, Yapong B, Kedjarune U. Occupational health problems of dentists in southern Thailand. Int Dent J Feb. 2000 50(1):36040.

17. Lopes AC, Almeida BK, Zanconato CM, Mondelli MFCG. Estudo dos limiares de audibilidade de altas frequências em crianças ouvintes com idades entre 7 e 13 anos. Dist. Comun. 2007, 19(2):173-180.

18. Lopes AC, Godoy JB. Considerações metodológicas para a investigação dos limiares de frequência ultra-altas em indivíduos expostos a o ruído ocupacional. Salusvita. Bauru. 2006, 25(2):149-160.

19. Luxon LM. The clinical diagnosis of noise induced hearing loss. Advances in Noise Research, Londres, 1998.

20. Matthews LJ, Lee FS, Mills Jh, Dubno JR. Extended highfrequency threshold in older adults. Journal of speech, language, and hearing research. 1997, 40:208-14.

21. Melo et al. Aspectos odontolegais da insalubridade na odontologia. RGO. Porto Alegre. 2008, 56(2):143-9.

22. Mota SSR. Estudo dos limiares auditivos de cirurgiões cirurgiões dentistas nas frequências de 500 a $16.000 \mathrm{~Hz}$.
Dissertação de Mestrado em Distúrbios da Comunicação. Curitiba: Universidade Tuiuti do Paraná, 2002.

23. Oliveira et al. Ruído ambiental e sua percepção pelos alunos de odontologia. Revista de Odontologia da UNESP. 2007, 36(1):9-16.

24. Áurea Otoni, Marlene Escher Boger, Anadergh BarbosaBranco, Helena Eri Shimizu, Mariluci Alves Maftum. Ruído ocupacional como fator de risco para perda auditiva. Cogitare Enferm. 2008, 13(3):367-73.

25. PAraguay ATT. Perda Auditiva Induzida por Ruído em Consultório Odontológico. Recife, 1999. Trabalho de conclusão de curso de especialização, CEFAC.

26. Porto MAA, Lopes AC, Gayva D, Lauris JRP . Avaliação da audição em frequências ultra-altas em indivíduos expostos ao ruído ocupacional. Pró-fono, Baueri-SP. 2004, 16(3):237250.

27. Presta AA, Garbin CAS, Garbin AJI, Saliba. Avaliação da Ocorrência de Doenças e Acidentes Ocupacionais entre Acadêmicos de Odontologia. Revista da Faculdade de Odontologia de Passo Fundo. 2004, 9(1):113-117.

28. Rabinowitz PM. Noise induced hearing loss. Am Fam Physician, Kansas City. 2000, 61(9):2749-56/2759-60.

29. Segurança e Medicina do Trabalho. Atlas S.A. 1991, 20 ed.; 16:95-6.

30. Sava LC, protocolo de avaliação para populações expostas a ruído indistrial. In: Morata TC, Zucki F. (Orgs). Caminhos para a saúde auditiva ambiental-ocupacional. São Paulo: Plexus. 2005, 189-202.

31. Silva IMC, Feitosa MAG. Audiometria de alta frequência em adultos jovens e mais velhos quando a audiometria convencional é normal. Rev. Bras. Otorrinolaringol. São Paulo. 72(5), Sept./Oct., 2006.

32. Souza HMMR. Ruído, o inimigo invisível: visão do cirurgião dentista. RBO, 54(2):97-101, 1997.

33. Tôrres BO, et al. A Perda Auditiva Induzida pelo Ruído (PAIR) na formação acadêmica: conhecimentos e medidas de prevenção - Odontologia. Clín. Científ., Recife. 2007, 6(2):151-154.

34. Zubick HH, Tolentino AT, Boffa J. Hearing loss and the high spped dental handpiece. Am J Public Health. 1980, 70(6):633-5. 